Draft VERSion MAY 1, 2020

Preprint typeset using $\mathrm{AT}_{\mathrm{E} X} \mathrm{X}$ style emulateapj v. 05/12/14

\title{
HIGH-VELOCITY TYPE IA SUPERNOVA HAS A UNIQUE HOST ENVIRONMENT
}

\author{
Yen-Chen PAN ${ }^{1,2}$ \\ Draft version May 1, 2020
}

\begin{abstract}
Ejecta velocity of type Ia supernovae (SNe Ia) is one powerful tool to differentiate between progenitor scenarios and explosion mechanisms. Here we revisit the relation between photospheric Si II $\lambda 6355$ velocities $\left(v_{\mathrm{Si} I}\right)$ and host-galaxy properties with $\sim 280 \mathrm{SNe}$ Ia. A more stringent criterion on the phase of SN spectra is adopted to classify SNe Ia in terms of their photospheric velocities. We find significant trend that SNe Ia with faster Si II $\lambda 6355$ (high- $v_{\mathrm{Si} \text { II }} \mathrm{SNe}$ Ia) tend to explode in massive environments, whereas their slower counterparts can be found in both lower-mass and massive environments. This trend is further supported by the direct measurements on host gas-phase metallicities. We suggest this relation is likely caused by at least two populations of SNe Ia. Since stars of higher metallicity (at a given mass) generally form less massive white dwarfs (WDs), our results support some theoretical models that high- $v_{\mathrm{Si}}$ SNe Ia may originate from sub-Chandrasekhar class of explosions. Previous observations also showed some evidence that high- $v_{\mathrm{Si} \text { II }}$ SNe Ia could be related to the single degenerate systems. However, we find high- $v_{\mathrm{Si} \text { II }} \mathrm{SNe}$ Ia do not come from particularly young populations. We conclude metallicity is likely the dominant factor in forming high- $v_{\mathrm{Si}}$ II $\mathrm{SNe}$ Ia. This also implies their potential evolution with redshift and impact on the precision of SN Ia cosmology.
\end{abstract}

\section{INTRODUCTION}

Type Ia supernovae ( $\mathrm{SNe}$ Ia) are believed to be the result of the thermonuclear explosion of an accreting carbon-oxygen white dwarf (WD) star in a close binary system (Nugent et al. 2011; Bloom et al. 2012). However, the nature of the companion star that donates material is not yet clear. The various possibilities include the single degenerate (Whelan \& Iben 1973) and double degenerate (Iben \& Tutukov 1984; Webbink 1984) scenarios, as well as more variations on these themes (for recent reviews, see Hillebrandt \& Niemeyer 2000, Hillebrandt et al. 2013 Maoz et al. 2014).

The host galaxies of SNe Ia has long been a profitable route to probe the SN Ia population, with the observed properties of SNe Ia known to correlate with the physical parameters of their host galaxies. Previous studies have found significant correlations between SN Ia light curve parameters and luminosities, and the properties of their host galaxies (e.g., Kelly et al. 2010 Lampeitl et al. 2010, Sullivan et al. 2010, D'Andrea et al. |2011; Johansson et al. 2013; Childress et al. 2013; Pan et al. 2014). SNe Ia with faster light curves are preferentially resided in massive and metal-rich galaxies than those in lower-mass and metal-poor systems. Galaxies with stronger star-formation and younger populations tend to host slower and brighter SNe Ia than passive and older galaxies.

Previous studies also showed some evidence that the SN Ia spectral features correlate with the host properties. By dividing SNe Ia into two sub-groups according to their photospheric velocities, Wang et al. (2013) found SNe Ia with high Si II $\lambda 6355$ velocities (high- $v_{\text {Si II }}$ SNe Ia, defined as $v_{\text {Si II }} \gtrsim 12,000 \mathrm{~km} \mathrm{~s}^{-1}$; Wang et al. 2009 ) tend to be more concentrated in the inner regions of their host galaxies, whereas the normal-velocity events

\footnotetext{
1 Division of Science, National Astronomical Observatory of Japan, 2-21-1 Osawa, Mitaka, Tokyo 181-8588, Japan

2 EACOA Fellow
}

(normal- $v_{\mathrm{Si} \text { II }}$ SNe Ia; defined as $v_{\mathrm{Si} \text { II }}<12,000 \mathrm{~km} \mathrm{~s}^{-1}$ ) span a wider range of radial distance. Given the metallicity gradients observed in both the Milky Way and many external galaxies (e.g., Henry \& Worthey 1999), they suggested the metallicity could be important in driving this relation. Pan et al. (2015, hereafter P15) further supported this idea by finding some evidence that high$v_{\mathrm{Si} \text { II }} \mathrm{SNe}$ Ia tend to reside in more massive galaxies than the normal- $v_{\mathrm{Si} I I}$ counterparts, although their results are not statistically significant due to the small sample size. These host studies together suggested there could be at least two distinct populations of SNe Ia in terms of their ejecta velocities.

High- $v_{\text {Si II }}$ SN Ia has long been suspected to have a different origin from normal- $v_{\text {SiII }} \mathrm{SN}$ Ia. This was firstly proposed by Wang et al. (2009), where they found high$v_{\text {Si II }} \mathrm{SNe}$ Ia tend to be redder and prefer a lower extinction ratio $\left(R_{V}\right)$ than normal- $v_{\text {Si II }}$ SNe Ia. Foley \& Kasen (2011) argued the high- $v_{\text {Si II }}$ SNe Ia actually do not have a different reddening law but are intrinsically redder than normal- $v_{\text {Si II }}$ SNe Ia. Recently, Wang et al. (2019) found some evidence that these high- $v_{\text {Si II }}$ SNe la tend to show blue excess in their late-time light-curves and variable $\mathrm{Na}$ I absorption lines in their spectra. These observations were attributed as the circumstellar dust surrounding the SNe. They claimed the high- $v_{\text {Si II }}$ SNe Ia are likely associated with the single degenerate systems.

Theoretical study also suggested the high- $v_{\text {Si II }}$ SNe Ia may originate from unique explosions. Using 1D WD explosion models, Polin et al. (2019) showed the subChandrasekhar explosions could produce SNe Ia with a wide range of $v_{\mathrm{Si} I I}$. Their results further indicated the high- $v_{\text {Si II }}$ SNe Ia could be primarily produced by subChandrasekhar type of explosions. The red color found for high- $v_{\text {Si II }}$ SNe Ia can also be explained by the line blanketing effect due the ashes of helium shell in their models.

In this paper, we revisit the relation between SN Ia 

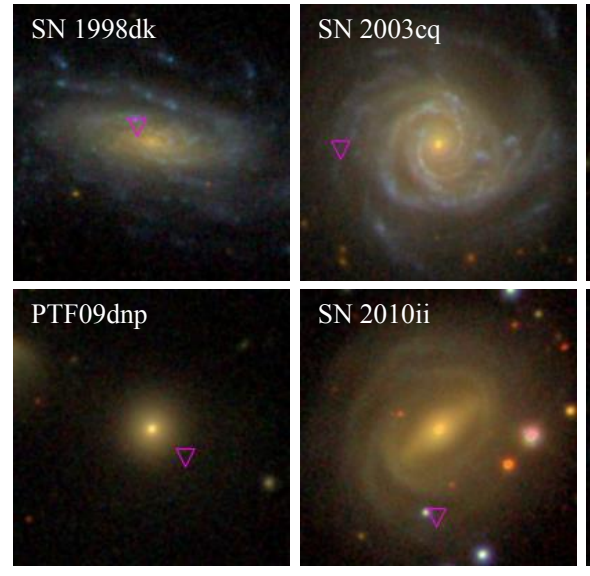
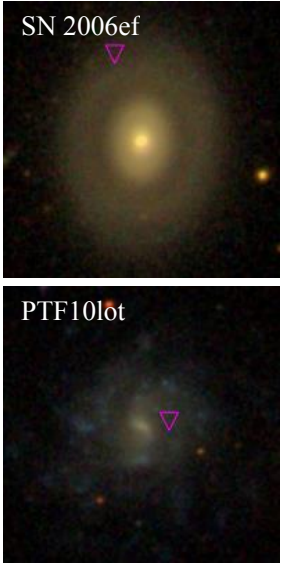
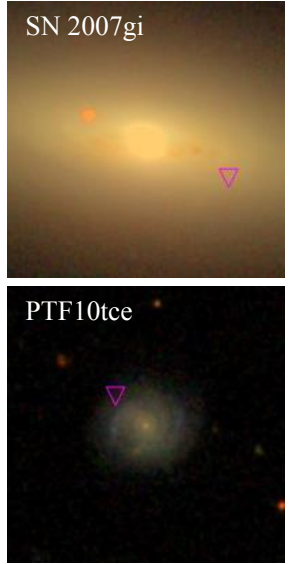
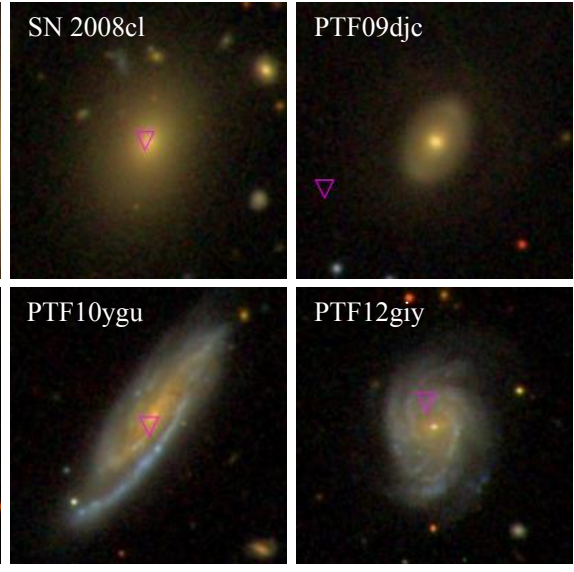

FIG. 1. - Some examples of high Si II $\lambda 6355$ velocity SN Ia (high- $v_{\text {Si II }}$ SN Ia; defined as $v_{\text {Si II }} \geq 12000 \mathrm{~km} \mathrm{~s}-1$ ) host galaxies in this work. The position of the SN is indicated by the purple triangle. Images are all generated from SDSS with a size of $80^{\prime \prime} \times 80^{\prime \prime}$. North is up and east is left.

$v_{\text {Si II }}$ and host-galaxy properties with a parent sample of $\sim 280$ SNe Ia ( $\gtrsim 2$ times larger than that in P15), with the purpose to differentiate the progenitor properties and explosion mechanisms between high- $v_{\text {Si II }}$ and normal- $v_{\text {Si II }}$ SNe Ia. A plan of the paper follows. In Section 2 we introduce our SN Ia spectral sample and the determination of host parameters. We show the results in Section 3 . The discussion and conclusions are presented in Section 4 and Section 5 respectively. Throughout this paper, we assume $\mathrm{H}_{0}=70 \mathrm{~km} \mathrm{~s}^{-1} \mathrm{Mpc}^{-1}$ and a flat universe with $\Omega_{\mathrm{M}}=0.3$.

\section{DATA AND METHOD}

\subsection{SN data}

In this work, we extend our analysis with the full spectroscopic sample studied in Maguire et al. (2014, hereafter M14). This is the parent sample of what was studied by P15, containing 264 spectroscopically normal SNe Ia with Si II $\lambda 6355$ measurements near peak (e.g., within 5 days from the peak luminosity). They were all discovered by the Palomar Transient Factory (PTF). In addition to the PTF sample, we further include the SNe studied in Silverman et al. (2015, hereafter S15) to increase our sample size. This added another $\sim 150 \mathrm{SNe}$ Ia with near-peak Si II $\lambda 6355$ measurements from the Berkeley SN Ia Program (BSNIP) after removing the duplicate objects from our PTF sample. This gives a sample of $\sim 400 \mathrm{SNe}$ Ia at $z<0.2$. The description of the spectroscopic observation and data reduction can be found in detail in M14 and S15.

Since M14 and S15 used very similar techniques in measuring the spectral features, we do not perform new measurements but simply adopting their results in our analysis. A complete description of the line measurement can be found in M14. Briefly speaking, the SN spectrum is firstly corrected into the rest frame, define (by eye inspection) continuum regions on either side of the feature, and fit a straight line pseudo-continuum across the absorption feature. The feature is then normalised by dividing it by the pseudo-continuum. A Gaussian fit is performed to the normalised Si II $\lambda 6355$ line in velocity space. The resulting fit then gives the velocity and pseudo-equivalent widths ( $\mathrm{pEW}$ ) of the feature.

\subsection{Host-galaxy properties}

The main purpose of this work is to investigate the relation between $v_{\mathrm{Si} \text { II }}$ of SNe Ia and their host properties. The host stellar mass $\left(M_{\text {stellar }}\right)$ is derived by fitting the photometry of the host galaxy with the photometric redshift code Z-PEG (Le Borgne \& Rocca-Volmerange 2002). The host photometry is provided from SDSS ugriz cata$\log$ (Abolfathi et al.2018). The SDSS model magnitudes are used here. Z-PEG fits the observed galaxy colours with galaxy spectral energy distribution (SED) templates corresponding to 9 spectral types (SB, Im, Sd, Sc, Sbc, $\mathrm{Sb}, \mathrm{Sa}, \mathrm{S} 0$, and E). Here we assume a Salpeter (1955) initial-mass function (IMF). The photometry is corrected for foreground Milky Way reddening with $R_{V}=3.1$ and a Cardelli, Clayton, \& Mathis (1989, CCM) reddening law. Fig. 1 shows some SDSS color images of high- $v_{\text {Si II }}$ SN Ia host galaxies studied in this work.

We also measure the host gas-phase metallicity and star-formation rate (SFR). This is done by obtaining the optical spectra of the host galaxies, primarily with the SDSS spectrograph on the Sloan Foundation 2.5-m telescope and Gemini Multi-Object Spectrographs (GMOS) on the Gemini Observatory. We fit the emission lines and stellar continuum of the host spectrum using the Interactive Data Language (IDL) codes PPXF (Cappellari \& Emsellem 2004) and GANDALF (Sarzi et al. 2006). A complete description of this process can be found in Pan et al. (2014). Briefly, PPXF fits the line-of-sight velocity distribution (LOSVD) of the stars in the galaxy in pixel space using a series of stellar templates. Before fitting the stellar continuum, the wavelengths of potential emission lines are masked to remove any possible contamination. The stellar templates are based on the MILES empirical stellar library (Sánchez-Blázquez et al. 2006; Vazdekis et al. 2010). A total of 288 templates is selected with $[M / H]=-1.71$ to +0.22 in 6 bins and ages ranging from 0.063 to 14.12 Gyr in 48 bins.

We correct all spectra for foreground Galactic reddening using the calibrations of Schlafly \& Finkbeiner (2011). The host-galaxy extinction is corrected with the two-component reddening model in GANDALF. The first component assumes a diffusive dust throughout the whole galaxy that affects the entire spectrum. It is determined by comparing the observed spectra to the un-reddened spectral templates. The second component measures the local dust around the nebular regions and 

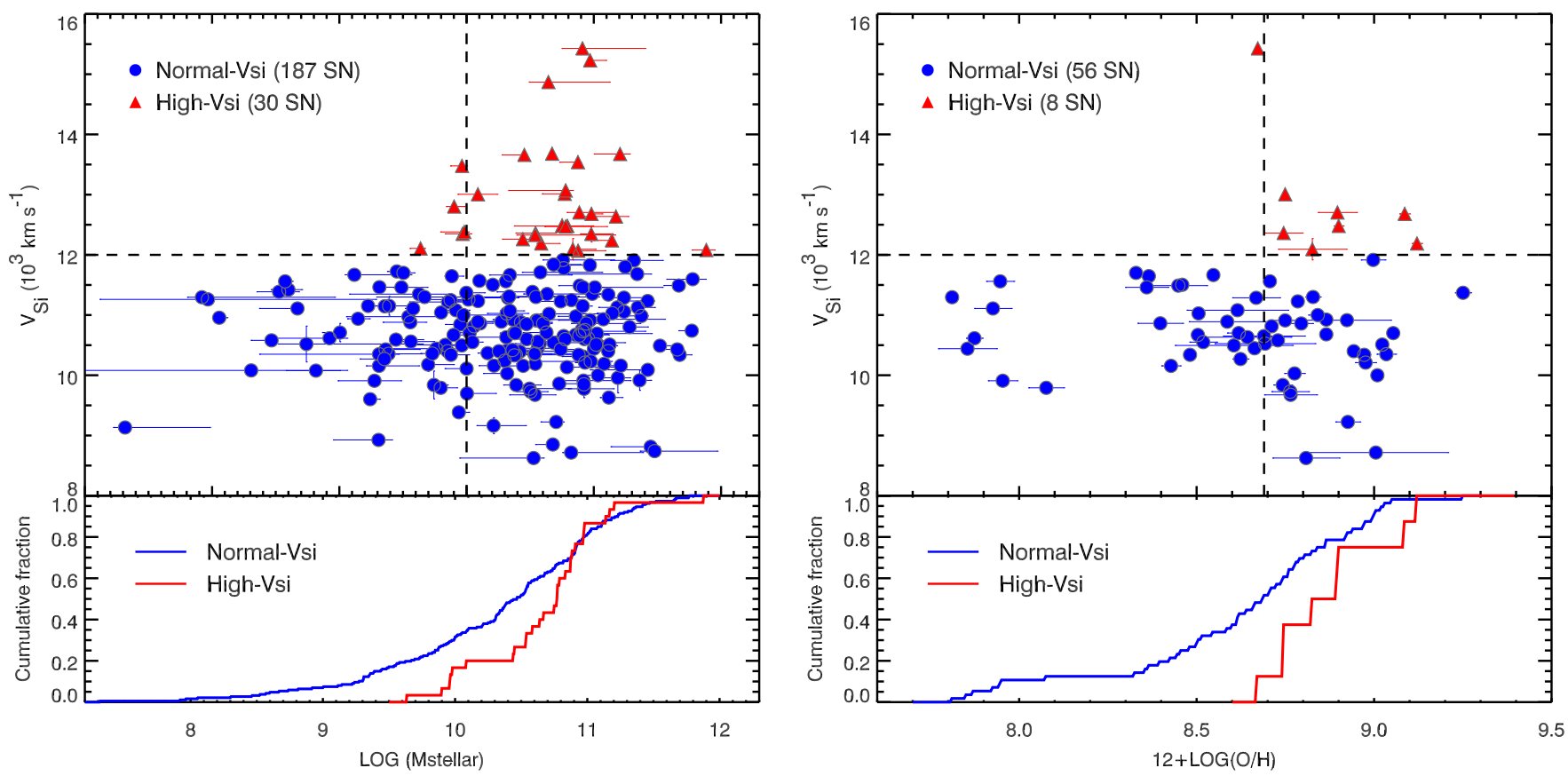

FIG. 2.- Left: The Si II $\lambda 6355$ velocities $\left(v_{\mathrm{Si} I I}\right)$ as a function of host-galaxy stellar mass $\left(M_{\mathrm{stellar}}\right)$. The high- $v_{\mathrm{Si} I I} \mathrm{SNe}$ Ia are shown as red triangles, and the normal- $v_{\mathrm{Si} \text { II }} \mathrm{SNe}$ Ia are shown as blue circles. The vertical and horizontal dashed lines represent the criterion used to split the sample in velocity and $M_{\text {stellar }}$ space, respectively. The bottom histograms show the cumulative fractions of $M_{\text {stellar }}$ for high- $v_{\mathrm{Si} \text { II }}$ and normal- $v_{\mathrm{Si} \text { II }} \mathrm{SNe}$ Ia. Right: The same as left panel, but with host-galaxy gas-phase metallicity instead.

affects only the emission lines. It is constrained only if the Balmer decrement (the $\mathrm{H} \alpha$ to $\mathrm{H} \beta$ line ratio) can be measured.

After the emission-line measurements from PPXF and GANDALF, we determine the SFR by adopting the conversion of Kennicutt (1998), which used evolutionary synthesis models to relate the luminosity of the $\mathrm{H} \alpha$ line to the SFR. We calculate the host gas-phase metallicity based on the diagnostics from Dopita et al. (2016). Dopita et al. (2016) used the ratios of $[\mathrm{N}$ II $] \lambda 6584$ to [S II] $\lambda \lambda 6717,6731$ and $[\mathrm{N}$ II] $\lambda 6584$ to $\mathrm{H} \alpha$ to calibrate the gas-phase metallicity. This has the advantage of requiring a narrow wavelength range and therefore is less affected by the reddening correction. We further use BPT diagrams (Baldwin, Phillips, \& Terlevich 1981) to check for potential contamination from active galactic nuclei (AGNs) in our host galaxies. The criteria proposed by Kewley et al. (2001) are adopted to distinguish between normal and AGN host galaxies. The potential AGNs are excluded from our emission line analyses. A summary of our measurements can be found in Table 1

\section{ANALYSIS}

We firstly investigate the relation between $v_{\mathrm{Si} I}$ and host $M_{\text {stellar. }}$ Ideally, one should distinguish between high- $v_{\text {Si II }}$ and normal- $v_{\text {Si II }}$ SNe Ia with the $v_{\text {Si II }}$ measured exactly at peak luminosity. In practice, previous studies generally used the spectra within a few days from the peak given that the spectral evolution is relatively mild at those epochs. M14 measured $v_{\mathrm{Si} \text { II }}$ using all the spectra observed within 5 days from the peak luminosity and did not apply phase corrections to their line measurements. Given a larger sample size, we adopt a more stringent phase criterion in this work; we use all the spectra observed within only 3 days from the peak luminosity. That way the $v_{\mathrm{Si} I I}$ of different $\mathrm{SNe}$ are compared at closer phases and reduces the uncertainties of phase evolution. This gives a final parent sample of $281 \mathrm{SNe}$ (41 of them are high- $v_{\mathrm{Si} I I} \mathrm{SNe}$ Ia), which is still more than 2 times larger than that studied in P15. Releasing this criterion does not change our conclusion but only making our results less significant.

The result is shown in the left panel of Fig. 2, We find clear evidence that most of the high- $v_{\mathrm{Si} I I}$ SNe Ia reside in massive galaxies $\left(\log \left(M_{\text {stellar }}\right)>10 M_{\odot}\right)$, whereas the normal- $v_{\mathrm{Si} \text { II }} \mathrm{SNe}$ Ia can be found in both lower-mass and massive galaxies. This is consistent with the finding in P15. Here we confirm this trend with a much larger sample and our results are statistically more significant. Both Kolmogorov-Smirnov (K-S) and Anderson-Darling (A-D) tests give a $p$-value of $\lesssim 0.02$ that the $M_{\text {stellar }}$ distributions of high- $v_{\mathrm{Si} I I}$ and normal- $v_{\mathrm{Si}}$ II $\mathrm{SNe}$ Ia are drawn from the same underlying population. This value is $\sim 10$ times smaller than that studied in P15. It is also evident that this relation is neither linear nor monotonic. In fact, it implies the existence of multiple populations of SNe Ia (see Section 4.1 for a discussion).

We also note the $v_{\mathrm{Si} \text { II }}$ of $\sim 12000 \mathrm{~km} \mathrm{~s}^{-1}$ is a fairly good criterion to distinguish between high- $v_{\mathrm{Si}}$ II and normal$v_{\text {Si II }}$ SNe Ia. For SNe with $v_{\text {Si II }}>12000 \mathrm{~km} \mathrm{~s}^{-1}, \sim 84$ percent of their host galaxies have $\log \left(M_{\text {stellar }}\right)>10 M_{\odot}$. The ratio goes up to only $\sim 90$ percent if the criterion is raised to $13000 \mathrm{~km} \mathrm{~s}^{-1}$, but drops significantly to $\sim 68$ percent if the criterion is lowered to $11000 \mathrm{~km} \mathrm{~s}^{-1}$. All of the SNe with $v_{\text {Si II }}>12000 \mathrm{~km} \mathrm{~s}^{-1}$ have $\log \left(M_{\text {stellar }}\right)>$ $9.6 M_{\odot}$.

Next we investigate the relation with host gas-phase metallicity. The result is shown in the right panel of Fig. 2. Given the tight relation between $M_{\text {stellar }}$ and metallicity (e.g., Tremonti et al. 2004), it is reasonable to suspect that the metallicity is the underlying source to drive the relation we see with $M_{\text {stellar}}$. In general, we find 
the relation between $v_{\mathrm{Si} \text { II }}$ and host metallicity is consistent with what we have found for host $M_{\text {stellar }}$. The host galaxies of high- $v_{\mathrm{Si} \text { II }} \mathrm{SNe}$ Ia tend to be metal-rich, having metallicities mostly above solar value (8.69; Allende Prieto et al. 2001). The K-S and A-D tests give a $p$-value of 0.05 and 0.04 , respectively, that the metallicity distributions of high- $v_{\text {Si II }}$ and normal- $v_{\text {Si II }}$ SNe Ia are drawn from the same underlying population. This is less significant than the trend with host $M_{\text {stellar }}$. However, our sample with host metallicity measurements is only one third the size of that with host $M_{\text {stellar }}$. The progenitor metallicity is also expected to differ from nuclear metallicity measurements performed in this work. A larger sample with direct metallicity measurements near the SN location is critical to constrain the metallicity effect in the future.

\section{DISCUSSION}

\subsection{Silicon velocity and metallicity}

Lentz et al. (2000) showed that the observed $v_{\mathrm{Si} \text { II }}$ could vary with the $\mathrm{C}+\mathrm{O}$ layer metallicity in $\mathrm{SN}$ Ia. The blue-shifted velocity of the Si II $\lambda 6355$ feature increase with $\mathrm{C}+\mathrm{O}$ layer metallicity due to the increasing opacity in the $\mathrm{C}+\mathrm{O}$ layer moving the features blueward and causing larger line velocities. P15 determined a linear relation between the $v_{\mathrm{Si} \text { II }}$ and $\mathrm{C}+\mathrm{O}$ metallicity of $\mathrm{SN}$ progenitor using the models of Lentz et al. (2000) and showed the $v_{\text {Si II }}$ increase with metallicities with a slope of $435 \mathrm{~km} \mathrm{~s}^{-1} \mathrm{dex}^{-1}$. They claimed the observed relation between host $M_{\text {stellar }}$ and $v_{\mathrm{SiII}}$ is in qualitative agreement with that of Lentz et al. (2000) models. However, fitting a linear relation between the observed $v_{\text {Si II }}$ and host gas-phase metallicity with our sample (i.e., right panel of Fig. 2 gives a slope of $87 \pm 427 \mathrm{~km} \mathrm{~s}^{-1} \mathrm{dex}^{-1}$, which is consistent with no trend.

In fact, it is now evident that both high- $v_{\text {SiII }}$ and normal- $v_{\text {Si II }}$ SNe Ia can be found in metal-rich host environments. If the high photospheric velocity is mainly caused by the increasing opacity (due to higher progenitor metallicity) in SN, we would expect a monotonic relation between $v_{\mathrm{Si} \text { II }}$ and host metallicity, instead of a L-shaped distribution shown in both panels of Fig. 2. Thus, it is not precise to say that the high- $v_{\mathrm{Si} \text { II }} \mathrm{SNe}$ Ia tend to reside in more metal-rich environments than that of normal- $v_{\mathrm{Si} \text { II }} \mathrm{SNe}$ Ia. While the opacity could still have some effect here, we argue that there are likely at least two populations of SNe Ia responsible for the observed trend. The high- $v_{\mathrm{Si} \text { II }} \mathrm{SNe}$ Ia could be part of a unique population which is sensitive to the progenitor metallicity and can only be formed in metal-rich environments (see Section 4.2 for a discussion).

\subsection{Implications on progenitor systems and explosion mechanisms}

Wang et al. (2013) found high- $v_{\text {Si II }}$ SNe Ia are more concentrated in the inner and brighter regions of their host galaxies. They suggested the high- $v_{\text {Si II }}$ SNe Ia likely originate from younger and more metal-rich progenitors than those of normal- $v_{\text {Si II }}$ SNe Ia. Recently, Wang et al. (2019) showed some evidence that these high- $v_{\text {Si II }}$ SNe Ia tend to present blue excess in their late-time light-curves and variable $\mathrm{Na}$ I absorption lines in the spectra. They attributed these observations to the circumstellar dust

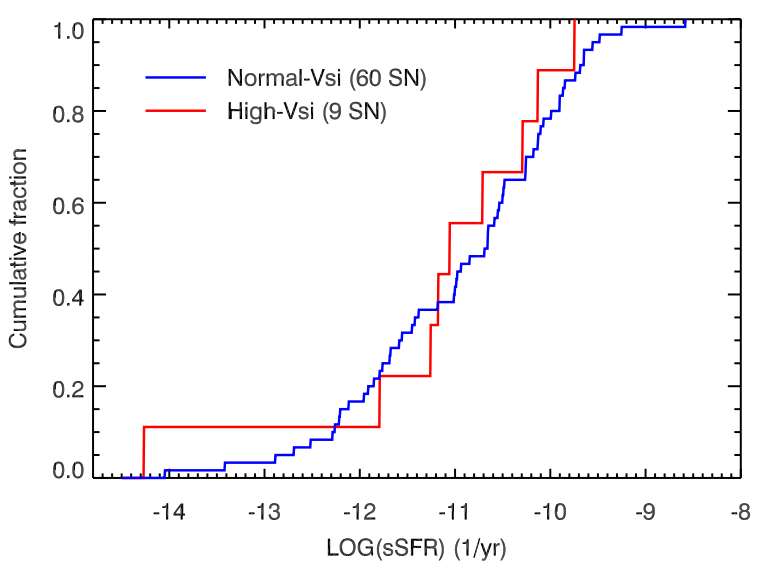

FIG. 3.- The cumulative fractions of specific star-formation rate (sSFR) for high- $v_{\text {Si II }}$ (red) and normal- $v_{\text {Si II }}$ (blue) SNe Ia.

surrounding the SNe and concluded that the high- $v_{\mathrm{Si}}$ II and normal- $v_{\mathrm{Si} I I} \mathrm{SNe}$ Ia are likely from the single degenerate and double degenerate systems, respectively.

Our results support that the high- $v_{\mathrm{Si} I I} \mathrm{SN}$ Ia has a strong preference to occur in metal-rich environment. However, we argue that they may not come from particularly young populations. Fig. 3 shows the cumulative fractions of specific star-formation rate (sSFR) for high$v_{\text {Si II }}$ and normal- $v_{\text {Si II }} \mathrm{SNe}$ Ia. Here the sSFR is defined as the SFR per unit $M_{\text {stellar. Theoretically, the sSFR }}$ is a more appropriate indicator to measure the relative star-formation activity of a galaxy as it measures the star-formation relative to the underlying galaxy stellar mass (Guzman et al. 1997). There is also a strong correlation between sSFR and age of the galaxies, in a sense that higher-sSFR galaxies tend to have younger stellar populations than lower-sSFR galaxies (e.g., Brinchmann et al. 2004).

We find the difference between high- $v_{\text {Si II }}$ and normal$v_{\text {Si II }} \mathrm{SNe}$ Ia is not statistically significant in terms of their host sSFR. The K-S test gives a $p$-value of 0.93 that the sSFR distributions of high- $v_{\text {Si II }}$ and normal- $v_{\text {Si II }}$ SNe Ia are drawn from the same underlying population. We determine a mean $\log (\mathrm{sSFR})$ of $-10.93 \pm 1.11 \mathrm{yr}^{-1}$ and $-11.15 \pm 1.16 \mathrm{yr}^{-1}$ for normal- $v_{\mathrm{Si} I I}$ and high- $v_{\mathrm{Si} I I}$ SNe Ia, respectively. Thus, the host galaxies of high- $v_{\text {Si II }}$ SNe Ia do not tend to be younger than their normal- $v_{\mathrm{Si} \text { II }}$ counterparts. This is consistent with the results in P15, where they showed the youngest populations are likely related to those SNe Ia with dispatched high-velocity features (HVFs), not those with high photospheric velocities. They also found there is a significant number (more than 30 percent) of high- $v_{\mathrm{Si}}$ II $\mathrm{SNe}$ Ia in early-type galaxies. Our results imply the metallicity is probably the only important (or dominant) factor in forming high$v_{\text {Si II }} \mathrm{SNe}$ Ia.

Theoretical studies also suggested the high- $v_{\text {Si II }}$ SNe Ia may have unique explosion mechanisms. Using 1D WD explosion models, Polin et al. (2019) showed the subChandrasekhar class of explosions can produce SNe Ia of a wide range of luminosities and photospheric velocities. In particular, their results indicated the high- $v_{\text {Si II }} \mathrm{SNe}$ Ia could be primarily produced by sub-Chandrasekhar explosions, whereas normal- $v_{\mathrm{Si} \text { II }} \mathrm{SNe}$ Ia can be produced by both sub-Chandrasekhar and near-Chandrasekhar explosions. The significant line blanketing due to the ashes 


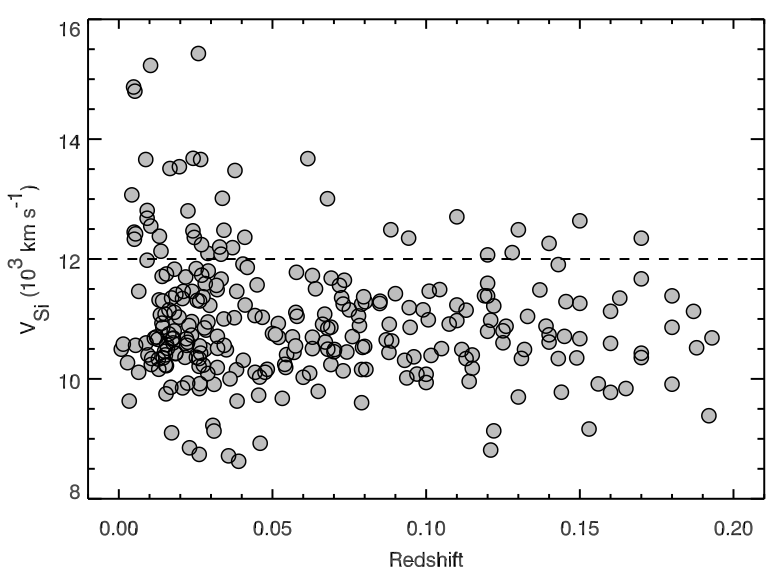

FIG. 4 . - The Si II $\lambda 6355$ velocities $\left(v_{\mathrm{Si} \text { II }}\right)$ as a function of redshift. The dashed line represents the the criterion used to split the high$v_{\text {Si II }}$ and normal- $v_{\text {Si II }}$ SNe Ia (i.e., $v_{\text {Si II }}=12000 \mathrm{~km} \mathrm{~s}^{-1}$ ).

of helium shell in their models also explained the intrinsically red color of high- $v_{\mathrm{Si} \text { II }}$ SNe Ia (e.g., Foley \& Kasen 2011). However, it is not yet clear the contribution of progenitor metallicity on such models.

Progenitor metallicity is believed to have significant impact on explosions of SN Ia. For example, it may affect the accretion onto the WD by changing the opacity in the wind for some single degenerate scenarios (e.g., Kobayashi et al. 1998). The mass of the WD is also expected to vary with metallicity. At a given mass, stars of higher metallicity generally produce less massive WDs (e.g., Umeda et al.1999). This implies they may be more difficult to reach the Chandrasekhar limit for explosions. Under the circumstances, the sub-Chandrasekhar class of explosions are probably more efficient and may account for some SNe Ia having higher progenitor metallicities. The preference of metal-rich environments can be used as a strong constraint to discriminate between models for high- $v_{\mathrm{Si} \text { II }} \mathrm{SNe}$ Ia in the future.

\subsection{Implications on cosmology}

Our results also have significant implications on cosmology. Foley \& Kasen (2011) found high- $v_{\mathrm{Si}}$ II SNe Ia are intrinsically redder (in terms of $B-V$ at maximum light) than normal- $v_{\mathrm{Si} \text { II }} \mathrm{SNe}$ Ia. By accounting for this color difference, they reduced the scatter in Hubble residuals (HRs). Also recently, Siebert et al. (2020) determined a $\sim 3-\sigma$ HR step between high- $v_{\mathrm{Si} \text { II }}$ and normal$v_{\mathrm{Si} \text { II }} \mathrm{SNe}$ Ia, with high- $v_{\mathrm{Si} \text { II }} \mathrm{SNe}$ Ia having more negative HRs than the normal- $v_{\mathrm{Si} I} \mathrm{SNe}$ Ia. These results are indicative that the ejecta velocity can be used to improve SN Ia distances.

Moreover, if the fraction of high- $v_{\mathrm{Si} I I}$ to normal- $v_{\mathrm{Si} \text { II }}$ $\mathrm{SNe}$ Ia changes with redshift, it could introduce significant bias on our cosmological analysis when assuming a single intrinsic color for SN Ia (Foley \& Kasen 2011). Given the strong preference of metal-rich environments for high- $v_{\mathrm{Si} \text { II }} \mathrm{SNe}$ Ia, we would expect an evolution on the number of discovered high- $v_{\mathrm{Si} \text { II }} \mathrm{SNe}$ Ia with redshift, with a decreased rate towards higher redshifts. Fig. 4 shows the $v_{\mathrm{Si} I}$ as the function of redshift with our sample. We find some evidence that the ratio of high- $v_{\text {Si II }}$ to normal- $v_{\mathrm{Si} I I} \mathrm{SNe}$ Ia tend to be higher at lower redshifts. At $z<0.1, \sim 16$ percent (13 percent if using untargeted PTF sample only) of SNe in our sample are high- $v_{\text {Si II }}$
$\mathrm{SNe}$, while only $\sim 11$ percent of the $\mathrm{SNe}$ are high- $v_{\mathrm{Si}}$ II $\mathrm{SNe}$ at $z>0.1$. It is also obvious that the extremely high- $v_{\text {Si II }}$ SNe Ia (e.g., $v_{\text {Si II }} \gtrsim 13000 \mathrm{~km} \mathrm{~s}^{-1}$ ) are hardly found at $z>0.1$.

However, the selection effect might play a role for $\mathrm{SNe}$ discovered at $z>0.1$ in our sample (for a discussion, see Pan et al. 2014). For example, we would expect a bias if the high- $v_{\mathrm{Si} \text { II }} \mathrm{SNe}$ Ia are significantly brighter or fainter than normal- $v_{\mathrm{Si} I I} \mathrm{SNe}$ Ia. Using $\Delta m_{15}(B)$ as the proxy of SN Ia brightness, we determine a mean $\Delta m_{15}(B)$ of $1.12 \pm 0.33 \mathrm{mag}$ and $1.12 \pm 0.22 \mathrm{mag}$ for normal- $v_{\mathrm{Si}}$ II and high- $v_{\text {Si II }}$ SNe Ia, respectively. This indicates (on average) they are not different in brightness. Thus, there is unlikely a Malmquist bias (at least) on the fraction of high- $v_{\text {Si II }}$ to normal- $v_{\text {Si II }}$ SNe Ia at higher redshifts. We also note that the high- $v_{\mathrm{Si} I} \mathrm{SNe}$ Ia tend to show less dispersion in $\Delta m_{15}(B)$ than that of normal- $v_{\mathrm{Si} \text { II }} \mathrm{SNe}$ Ia. It is unclear if this trend is intrinsic to the explosion or simply due to the smaller sample size of high- $v_{\mathrm{Si}}$ II $\mathrm{SNe}$ Ia.

Another caveat is the difficulty in finding SNe on very bright galaxy backgrounds, where the contrast of the SN over the host galaxy is low. Since high- $v_{\mathrm{Si}}$ II $\mathrm{SNe}$ Ia tend to be found in massive galaxies and are more concentrated in the inner regions of their hosts, they may be more difficult to be fond at higher redshifts. However, this is only an issue with modern image subtraction techniques when the SN brightness drops to $<10 \%$ of that of the host background (e.g., Perrett et al. 2010). The future analysis with data taken from higher-redshift surveys (e.g., Pan-STARRS1; Pan et al., in preparation) will be necessary to constrain the potential evolution of high- $v_{\text {Si II }}$ SNe Ia.

\section{CONCLUSIONS}

In this work, we investigate the relation between photospheric Si II $\lambda 6355$ velocities $\left(v_{\mathrm{Si} \text { II }}\right)$ and host-galaxy properties of SN Ia. A more stringent criterion on the phase of SN spectra is adopted to distinguish between normal$v_{\mathrm{Si} I I}$ and high- $v_{\mathrm{Si} \text { II }}$ SNe Ia. We find the high- $v_{\mathrm{Si} \text { II }} \mathrm{SNe}$ Ia are likely formed from a distinct population which favors massive host environments. This is further supported by the direct measurements on host gas-phase metallicities. Although opacity may have some effect (due to progenitor metallicity), we argue the difference in photospheric velocities between high- $v_{\mathrm{Si} \text { II }}$ and normal- $v_{\mathrm{Si} \text { II }} \mathrm{SNe}$ Ia is mainly caused by different explosion mechanisms.

Theoretical studies suggested the high- $v_{\mathrm{Si} \text { II }} \mathrm{SNe}$ Ia may originate from sub-Chandrasekhar explosions. This is consistent with our results. At a given mass, stars of higher metallicities generally produce less massive WDs. This may increase the chance for them to explode under sub-Chandrasekhar mass. Nevertheless, the detailed investigation is still needed to evaluate the effect of progenitor metallicity on such models.

Previous studies also suggested the high- $v_{\mathrm{Si} \text { II }}$ and normal- $v_{\mathrm{Si} \text { II }} \mathrm{SNe}$ Ia could be formed via single degenerate and double degenerate scenarios, respectively. However, we find high- $v_{\mathrm{Si} I I} \mathrm{SNe}$ Ia do not tend to originate from younger populations than that of normal- $v_{\text {Si II }}$ $\mathrm{SNe}$ Ia. We argue the metallicity is the only important factor in forming high- $v_{\mathrm{Si} I I} \mathrm{SNe}$ Ia.

Our results also imply potential evolution of high- $v_{\text {Si II }}$ $\mathrm{SNe}$ Ia. We would expect less high- $v_{\mathrm{Si} \text { II }} \mathrm{SNe}$ Ia to be discovered at higher redshifts when the Universe is more 
metal-poor than present. This evolution could introduce a bias on our cosmological analysis given that high- $v_{\text {Si II }}$ and normal- $v_{\mathrm{Si} \text { II }} \mathrm{SNe}$ Ia tend to have intrinsically different colors. Future spectroscopic studies using higherredshift dataset will be critical to measure this evolution effect.

\section{ACKNOWLEDGMENTS}

Y.-C.P. is supported by the East Asian Core Observatories Association (EACOA) Fellowship.

\section{REFERENCES}

Abolfathi, B., Aguado, D. S., Aguilar, G., et al. 2018, ApJS, 235, 42

Allende Prieto, C., Lambert, D. L., \& Asplund, M. 2001, ApJ, 556, L63

Baldwin, J. A., Phillips, M. M., \& Terlevich, R. 1981, PASP, 93, 5

Bloom, J. S., Kasen, D., Shen, K. J., et al. 2012, ApJ, 744, L17

Brinchmann, J., Charlot, S., White, S. D. M., et al. 2004, MNRAS, 351, 1151

Cappellari, M., \& Emsellem, E. 2004, PASP, 116, 138

Cardelli, J. A., Clayton, G. C., \& Mathis, J. S. 1989, ApJ, 345, 245

Childress, M., Aldering, G., Antilogus, P., et al. 2013, ApJ, 770, 108

D'Andrea, C. B., Gupta, R. R., Sako, M., et al. 2011, ApJ, 743, 172

Dopita, M. A., Kewley, L. J., Sutherland, R. S., \& Nicholls, D. C. 2016, Ap\&SS, 361, 61

Foley, R. J., \& Kasen, D. 2011, ApJ, 729, 55

Guzman, R., Gallego, J., Koo, D. C., et al. 1997, ApJ, 489, 559

Henry, R. B. C., \& Worthey, G. 1999, PASP, 111, 919

Hillebrandt, W., Kromer, M., Röpke, F. K., \& Ruiter, A. J. 2013 Frontiers of Physics, 8, 116

Hillebrandt, W., \& Niemeyer, J. C. 2000, ARA\&A, 38, 191

Iben, Jr., I., \& Tutukov, A. V. 1984, ApJS, 54, 335

Johansson, J., Thomas, D., Pforr, J., et al. 2013, MNRAS, 435, 1680

Kelly, P. L., Hicken, M., Burke, D. L., Mandel, K. S., \& Kirshner, R. P. 2010, ApJ, 715, 743

Kennicutt, J. R. C. 1998, ARA\&A, 36, 189

Kewley, L. J., Dopita, M. A., Sutherland, R. S., Heisler, C. A., \& Trevena, J. 2001, ApJ, 556, 121

Kobayashi, C., Tsujimoto, T., Nomoto, K., Hachisu, I., \& Kato, M. 1998, ApJ, 503, L155

Lampeitl, H., Smith, M., Nichol, R. C., et al. 2010, ApJ, 722, 566

Le Borgne, D., \& Rocca-Volmerange, B. 2002, A\&A, 386, 446

Lentz, E. J., Baron, E., Branch, D., Hauschildt, P. H., \& Nugent, P. E. 2000, ApJ, 530, 966
Maguire, K., Sullivan, M., Pan, Y. C., et al. 2014, MNRAS, 444, 3258

Maoz, D., Mannucci, F., \& Nelemans, G. 2014, ARA\&A, 52, 107

Nugent, P. E., Sullivan, M., Cenko, S. B., et al. 2011, Nature, 480,344

Pan, Y. C., Sullivan, M., Maguire, K., et al. 2015, MNRAS, 446, 354

Pan, Y.-C., Sullivan, M., Maguire, K., et al. 2014, MNRAS, 438, 1391

Perrett, K., Balam, D., Sullivan, M., et al. 2010, AJ, 140, 518

Polin, A., Nugent, P., \& Kasen, D. 2019, ApJ, 873, 84

Salpeter, E. E. 1955, ApJ, 121, 161

Sánchez-Blázquez, P., Peletier, R. F., Jiménez-Vicente, J., et al. 2006, MNRAS, 371, 703

Sarzi, M., Falcón-Barroso, J., Davies, R. L., et al. 2006, MNRAS, 366,1151

Schlafly, E. F., \& Finkbeiner, D. P. 2011, ApJ, 737, 103

Siebert, M. R., Foley, R. J., Jones, D. O., \& Davis, K. W. 2020, MNRAS, arXiv:2002.09490

Silverman, J. M., Vinkó, J., Marion, G. H., et al. 2015, MNRAS, 451, 1973

Sullivan, M., Conley, A., Howell, D. A., et al. 2010, MNRAS, 406, 782

Tremonti, C. A., Heckman, T. M., Kauffmann, G., et al. 2004, ApJ, 613, 898

Umeda, H., Nomoto, K., Yamaoka, H., \& Wanajo, S. 1999, ApJ, 513,861

Vazdekis, A., Sánchez-Blázquez, P., Falcón-Barroso, J., et al. 2010, MNRAS, 404, 1639

Wang, X., Chen, J., Wang, L., et al. 2019, ApJ, 882, 120

Wang, X., Wang, L., Filippenko, A. V., Zhang, T., \& Zhao, X. 2013, Science, 340, 170

Wang, X., Filippenko, A. V., Ganeshalingam, M., et al. 2009, ApJ, 699, L139

Webbink, R. F. 1984, ApJ, 277, 355

Whelan, J., \& Iben, Jr., I. 1973, ApJ, 186, 1007 
TABLE 1

SUMMARY OF OUR SAMPLE IN THIS WORK.

\begin{tabular}{|c|c|c|c|c|}
\hline SN Name & Redshift & $\begin{array}{c}v_{\mathrm{Si} I I} \\
\left(\mathrm{~km} \mathrm{~s}^{-1}\right)\end{array}$ & $\begin{array}{c}\log M_{\text {stellar }} \\
\left(\mathrm{M}_{\odot}\right)\end{array}$ & $12+\log (\mathrm{O} / \mathrm{H})$ \\
\hline PTF09bai & 0.180 & $11385 \pm 18$ & $10.518_{-0.279}^{+0.104}$ & $\cdots$ \\
\hline PTF09bj & 0.144 & $9777 \pm 50$ & $10.495_{-0.123}^{+0.324}$ & $\ldots$ \\
\hline PTF09djc & 0.034 & $13013 \pm 16$ & $10.771_{-0.176}^{+0.1008}$ & $\ldots$ \\
\hline PTF09dlc & 0.067 & $10615 \pm 9$ & $8.923_{-0.433}^{+0.138}$ & $7.874 \pm 0.023$ \\
\hline PTF09dnl & 0.024 & $10955 \pm 4$ & $8.056_{-0.053}^{+0.062}$ & $\ldots$ \\
\hline PTF09dnp & 0.037 & $12189 \pm 7$ & $10.586_{-0.033}^{+0.143}$ & $9.121 \pm 0.013$ \\
\hline PTF09dqt & 0.113 & $11146 \pm 22$ & $9.357_{-0.011}^{+0.055}$ & $\ldots$ \\
\hline PTF09dxo & 0.052 & $10924 \pm 10$ & $10.364_{-0.157}^{+0.449}$ & $8.865 \pm 0.014$ \\
\hline PTF09e & 0.149 & $10349 \pm 38$ & $9.312_{-0.939}^{+0.154}$ & $\ldots$ \\
\hline PTF09fox & 0.072 & $11560 \pm 11$ & $10.309_{-0.034}^{+0.033}$ & $8.706 \pm 0.005$ \\
\hline PTF09foz & 0.054 & $10190 \pm 8$ & $10.540_{-0.147}^{+0.039}$ & $\ldots$ \\
\hline PTF09gn & 0.139 & $10880 \pm 72$ & $9.558_{-0.215}^{+0.124}$ & $\ldots$ \\
\hline PTF09gul & 0.072 & $11352 \pm 33$ & $10.988_{-0.011}^{+0.045}$ & \\
\hline PTF09h & 0.121 & $10979 \pm 31$ & $10.859_{-0.140}^{+0.074}$ & $\cdots$ \\
\hline PTF09ib & 0.122 & $11216 \pm 15$ & $9.854_{-0.303}^{+0.177}$ & $\cdots$ \\
\hline PTF09isn & 0.101 & $11463 \pm 37$ & $9.315_{-0.015}^{+0.128}$ & $\ldots$ \\
\hline PTF09s & 0.046 & $8926 \pm 14$ & $9.308_{-0.355}^{+0.104}$ & $\ldots$ \\
\hline PTF09v & 0.119 & $11386 \pm 37$ & $8.526_{-0.173}^{+0.154}$ & $\cdots$ \\
\hline PTF10aaea & 0.160 & $9775 \pm 147$ & $10.923_{-0.125}^{+0.232}$ & $\ldots$ \\
\hline PTF10abjv & 0.076 & $10705 \pm 27$ & $10.024_{-0.079}^{+0.039}$ & $\ldots$ \\
\hline PTF10acqp & 0.170 & $12347 \pm 132$ & $10.980_{-0.085}^{+0.167}$ & $\ldots$ \\
\hline PTF10bhw & 0.110 & $11233 \pm 25$ & $11.423_{-0.239}^{+0.049}$ & $\ldots$ \\
\hline PTF10cmj & 0.112 & $10490 \pm 30$ & $11.117_{-0.057}^{+0.064}$ & $\ldots$ \\
\hline PTF10cwm & 0.079 & $10525 \pm 18$ & $10.575_{-0.194}^{+0.072}$ & $8.693 \pm 0.141$ \\
\hline PTF10cxk & 0.018 & $10938 \pm 13$ & $9.147_{-0.083}^{+0.090}$ & $\ldots$ \\
\hline PTF10duy & 0.079 & $9602 \pm 15$ & $9.242_{-0.047}^{+0.073}$ & $\ldots$ \\
\hline PTF10duz & 0.064 & $11502 \pm 33$ & $10.206_{-0.044}^{+0.111}$ & $8.459 \pm 0.010$ \\
\hline PTF10fej & 0.110 & $12703 \pm 82$ & $10.886_{-0.096}^{+0.270}$ & $8.897 \pm 0.054$ \\
\hline PTF10fj & 0.050 & $10757 \pm 19$ & $10.906_{-0.009}^{+0.137}$ & $\ldots$ \\
\hline PTF10fxe & 0.099 & $11152 \pm 155$ & $9.391_{-0.149}^{+0.206}$ & $\ldots$ \\
\hline PTF10fxl & 0.030 & $11227 \pm 12$ & $10.740_{-0.165}^{+0.013}$ & $8.785 \pm 0.003$ \\
\hline PTF10fxp & 0.104 & $11490 \pm 17$ & $11.668_{-0.117}^{+0.039}$ & $\ldots$ \\
\hline PTF10fxq & 0.107 & $10914 \pm 34$ & $10.971_{-0.249}^{+0.079}$ & $8.923 \pm 0.123$ \\
\hline PTF10fyl & 0.055 & $10405 \pm 9$ & $10.312_{-0.180}^{+0.004}$ & $\ldots$ \\
\hline PTF10glo & 0.075 & $11151 \pm 31$ & $9.226_{-0.029}^{+0.083}$ & $\ldots$ \\
\hline PTF10gnj & 0.078 & $10889 \pm 19$ & $10.273_{-0.120}^{+0.098}$ & $8.586 \pm 0.107$ \\
\hline PTF10goo & 0.087 & $10651 \pm 31$ & $10.763_{-0.210}^{+0.106}$ & $8.690 \pm 0.114$ \\
\hline PTF10gop & 0.097 & $10080 \pm 33$ & $8.307_{-1.440}^{+0.753}$ & $\ldots$ \\
\hline PTF10goq & 0.088 & $10910 \pm 59$ & $10.557_{-0.215}^{+0.086}$ & $\ldots$ \\
\hline PTF10hdm & 0.165 & $9839 \pm 230$ & $9.743_{-0.030}^{+0.106}$ & $\ldots$ \\
\hline PTF10hld & 0.038 & $13477 \pm 24$ & $9.964_{-0.090}^{+0.037}$ & $\cdots$ \\
\hline PTF10jab & 0.187 & $11126 \pm 16$ & $11.346_{-0.014}^{+0.132}$ & $\ldots$ \\
\hline PTF10lot & 0.022 & $12802 \pm 16$ & $9.902_{-0.061}^{+0.079}$ & $\ldots$ \\
\hline PTF10mtd & 0.079 & $11248 \pm 15$ & $9.875_{-0.065}^{+0.101}$ & $\ldots$ \\
\hline PTF10mwb & 0.031 & $9909 \pm 4$ & $9.276_{-0.286}^{+0.208}$ & $7.954 \pm 0.041$ \\
\hline PTF10ncy & 0.130 & $9697 \pm 45$ & $10.003_{-0.031}^{+0.226}$ & $\ldots$ \\
\hline PTF10ncz & 0.170 & $10429 \pm 95$ & $9.364_{-0.025}^{+0.266}$ & $\cdots$ \\
\hline PTF10nda & 0.101 & $10985 \pm 26$ & $11.374_{-0.216}^{+0.049}$ & $\ldots$ \\
\hline PTF10nhu & 0.153 & $9163 \pm 132$ & $10.212_{-0.131}^{+0.252}$ & $\ldots$ \\
\hline PTF10nnh & 0.150 & $10670 \pm 106$ & $10.856_{-0.065}^{+0.261}$ & $\cdots$ \\
\hline PTF10nvh & 0.068 & $10843 \pm 8$ & $9.950_{-0.117}^{+0.071}$ & $\ldots$ \\
\hline PTF10oth & 0.145 & $11289 \pm 35$ & $11.237_{-0.103}^{+0.174}$ & $\ldots$ \\
\hline PTF10pvh & 0.105 & $10504 \pm 39$ & $9.833_{-0.247}^{+0.179}$ & $\cdots$ \\
\hline PTF10pvi & 0.080 & $11286 \pm 46$ & $10.326_{-0.178}^{+0.079}$ & $8.668 \pm 0.066$ \\
\hline PTF10qhp & 0.032 & $11488 \pm 25$ & $10.889_{-0.165}^{+0.025}$ & $8.448 \pm 0.080$ \\
\hline PTF10qj1 & 0.058 & $11109 \pm 5$ & $8.671_{-0.299}^{+0.020}$ & $7.926 \pm 0.052$ \\
\hline PTF10qjq & 0.028 & $10813 \pm 12$ & $10.059_{-0.044}^{+0.087}$ & $8.711 \pm 0.003$ \\
\hline
\end{tabular}


TABLE 2

SumMary OF OUR SAMPLE IN THIS WORK (CONTINUED).

\begin{tabular}{|c|c|c|c|c|}
\hline SN Name & Redshift & $\begin{array}{c}v_{\mathrm{Si} I I} \\
\left(\mathrm{~km} \mathrm{~s}^{-1}\right)\end{array}$ & $\begin{array}{c}\log M_{\text {stellar }} \\
\left(\mathrm{M}_{\odot}\right)\end{array}$ & $12+\log (\mathrm{O} / \mathrm{H})$ \\
\hline PTF10qkf & 0.080 & $10156 \pm 55$ & $10.445_{-0.161}^{+0.115}$ & $8.428 \pm 0.026$ \\
\hline PTF10qky & 0.074 & $10448 \pm 13$ & $10.559_{-0.116}^{+0.094}$ & $8.663 \pm 0.005$ \\
\hline PTF10qny & 0.033 & $11664 \pm 12$ & $10.340_{-0.160}^{+0.484}$ & $8.547 \pm 0.014$ \\
\hline PTF10qsc & 0.088 & $10441 \pm 31$ & $9.762_{-0.378}^{+0.137}$ & $7.854 \pm 0.082$ \\
\hline PTF10qwg & 0.068 & $13006 \pm 16$ & $10.089_{-0.157}^{+0.151}$ & $8.749 \pm 0.006$ \\
\hline PTF10qyq & 0.160 & $10592 \pm 99$ & $9.445_{-0.050}^{+0.212}$ & $\ldots$ \\
\hline PTF10rab & 0.085 & $11296 \pm 70$ & $7.920_{-0.114}^{+1.043}$ & $7.811 \pm 0.005$ \\
\hline PTF10ran & 0.160 & $11127 \pm 57$ & $11.185_{-0.147}^{+0.088}$ & $\ldots$ \\
\hline PTF10rhi & 0.085 & $11257 \pm 35$ & $10.014_{-0.364}^{+0.077}$ & $\cdots$ \\
\hline PTF10tce & 0.041 & $12362 \pm 12$ & $10.542_{-0.155}^{+0.161}$ & $8.745 \pm 0.053$ \\
\hline PTF10tqy & 0.045 & $11568 \pm 11$ & $10.101_{-0.005}^{+0.162}$ & $\ldots$ \\
\hline PTF10trs & 0.073 & $11248 \pm 2$ & $10.824_{-0.070}^{+0.062}$ & $\cdots$ \\
\hline PTF10trw & 0.170 & $10355 \pm 40$ & $9.387_{-0.024}^{+0.259}$ & $\cdots$ \\
\hline PTF10twd & 0.073 & $11646 \pm 23$ & $9.884_{-0.055}^{+0.089}$ & $8.365 \pm 0.006$ \\
\hline PTF10ucl & 0.080 & $10541 \pm 15$ & $10.683_{-0.020}^{+0.561}$ & $\ldots$ \\
\hline PTF10ufj & 0.073 & $10134 \pm 10$ & $10.789_{-0.051}^{+0.428}$ & $\ldots$ \\
\hline PTF10urn & 0.110 & $10972 \pm 76$ & $9.543_{-0.487}^{+0.262}$ & $\cdots$ \\
\hline PTF10vfo & 0.088 & $12488 \pm 63$ & $10.751_{-0.022}^{+0.011}$ & $\cdots$ \\
\hline PTF10viq & 0.034 & $12480 \pm 10$ & $10.792_{-0.417}^{+0.201}$ & $8.900 \pm 0.013$ \\
\hline PTF10wnm & 0.066 & $10910 \pm 11$ & $10.548_{-0.045}^{+0.045}$ & $8.749 \pm 0.044$ \\
\hline PTF10wnq & 0.069 & $11681 \pm 56$ & $11.341_{-0.543}^{+0.107}$ & $\ldots$ \\
\hline PTF10wov & 0.096 & $10368 \pm 27$ & $10.165_{-0.076}^{+0.276}$ & $\ldots$ \\
\hline PTF10wri & 0.120 & $11595 \pm 20$ & $11.777_{-0.204}^{+0.101}$ & $\cdots$ \\
\hline PTF10xeb & 0.122 & $9132 \pm 62$ & $7.316_{-0.091}^{+0.665}$ & $\cdots$ \\
\hline PTF10xir & 0.052 & $10692 \pm 19$ & $11.025_{-0.178}^{+0.428}$ & $\cdots$ \\
\hline PTF10xtp & 0.102 & $10391 \pm 103$ & $10.419_{-0.133}^{+0.099}$ & $\cdots$ \\
\hline PTF10ygr & 0.115 & $10177 \pm 114$ & $9.698_{-0.022}^{+0.200}$ & $\cdots$ \\
\hline PTF10ygu & 0.026 & $15428 \pm 11$ & $10.911_{-0.161}^{+0.491}$ & $8.672 \pm 0.003$ \\
\hline PTF10yux & 0.058 & $11777 \pm 19$ & $10.764_{-0.066}^{+0.006}$ & $\ldots$ \\
\hline PTF10zai & 0.036 & $8715 \pm 59$ & $10.822_{-0.067}^{+0.563}$ & $9.005 \pm 0.203$ \\
\hline PTF10zak & 0.040 & $11915 \pm 10$ & $10.757_{-0.157}^{+0.400}$ & $8.997 \pm 0.034$ \\
\hline PTF10zbn & 0.114 & $9957 \pm 249$ & $11.189_{-0.160}^{+0.094}$ & $\ldots$ \\
\hline PTF10zgy & 0.044 & $11056 \pm 40$ & $11.238_{-0.000}^{+0.000}$ & $\cdots$ \\
\hline PTF11apk & 0.041 & $10310 \pm 10$ & $10.914_{-0.164}^{+0.007}$ & $\cdots$ \\
\hline PTF11bjk & 0.140 & $10613 \pm 61$ & $10.954_{-0.203}^{+0.064}$ & $\ldots$ \\
\hline PTF11blu & 0.068 & $10495 \pm 35$ & $9.963_{-0.081}^{+0.016}$ & $8.605 \pm 0.165$ \\
\hline PTF11byi & 0.039 & $8625 \pm 25$ & $10.527_{-0.581}^{+0.077}$ & $8.808 \pm 0.094$ \\
\hline PTF11ctn & 0.079 & $10156 \pm 11$ & $9.312_{-0.100}^{+0.313}$ & $\ldots$ \\
\hline PTF11cyv & 0.115 & $10398 \pm 46$ & $9.851_{-0.095}^{+0.105}$ & $\cdots$ \\
\hline PTF11deg & 0.063 & $11724 \pm 31$ & $9.452_{-0.0}^{+0 . c}$ & $\cdots$ \\
\hline PTF11dws & 0.150 & $12637 \pm 45$ & $11.174_{-0.308}^{+0.095}$ & $\cdots$ \\
\hline PTF11dzm & 0.041 & $11232 \pm 59$ & $10.090_{-0.163}^{+0.007}$ & $\cdots$ \\
\hline PTF11eot & 0.090 & $11421 \pm 50$ & $8.600_{-0.135}^{+0.105}$ & $\cdots$ \\
\hline PTF11fjw & 0.193 & $10684 \pm 66$ & $10.892_{-0.136}^{+0.216}$ & $\cdots$ \\
\hline PTF11for & 0.128 & $12106 \pm 83$ & $9.638_{-0.083}^{+0.033}$ & $\ldots$ \\
\hline PTF11gdh & 0.026 & $9838 \pm 13$ & $10.390_{-0.099}^{+0.190}$ & $8.743 \pm 0.025$ \\
\hline PTF11gin & 0.163 & $11347 \pm 42$ & $9.626_{-0.546}^{+0.255}$ & $\ldots$ \\
\hline PTF11gjb & 0.125 & $10601 \pm 42$ & $10.926_{-0.131}^{+0.052}$ & $\ldots$ \\
\hline PTF11glq & 0.156 & $9916 \pm 166$ & $11.360_{-0.019}^{+0.088}$ & $\cdots$ \\
\hline PTF11gnj & 0.131 & $10341 \pm 61$ & $10.387_{-0.032}^{+0.114}$ & $\ldots$ \\
\hline PTF11khk & 0.031 & $9224 \pm 11$ & $10.704_{-0.120}^{+0.058}$ & $8.926 \pm 0.034$ \\
\hline PTF11kod & 0.188 & $10519 \pm 297$ & $8.741_{-0.220}^{+0.912}$ & $\ldots$ \\
\hline PTF11kpb & 0.143 & $10339 \pm 19$ & $11.675_{-0.014}^{+0.091}$ & $\cdots$ \\
\hline PTF11kqm & 0.126 & $10878 \pm 21$ & $10.428_{-0.030}^{+0.241}$ & $\ldots$ \\
\hline PTF11kx & 0.047 & $11027 \pm 40$ & $\begin{array}{r}10.315_{-0.152}^{+0.169} \\
\end{array}$ & $8.505 \pm 0.007$ \\
\hline PTF11lbc & 0.150 & $11260 \pm 44$ & $7.967_{-0.848}^{+1.320}$ & $\ldots$ \\
\hline PTF11lmz & 0.061 & $13674 \pm 21$ & $11.206_{-0.203}^{+0.074}$ & $\cdots$ \\
\hline
\end{tabular}


TABLE 3

Summary OF OUR SAMPLE IN THIS WORK (CONTINUED).

\begin{tabular}{|c|c|c|c|c|}
\hline SN Name & Redshift & $\begin{array}{c}v_{\mathrm{Si} \mathrm{II}} \\
\left(\mathrm{km} \mathrm{s}^{-1}\right)\end{array}$ & $\begin{array}{c}\log M_{\text {stellar }} \\
\left(\mathrm{M}_{\odot}\right)\end{array}$ & $12+\log (\mathrm{O} / \mathrm{H})$ \\
\hline PTF11opu & 0.065 & $9791 \pm 47$ & $9.797_{-0.094}^{+0.127}$ & $8.076 \pm 0.050$ \\
\hline PTF11qpc & 0.089 & $10632 \pm 23$ & $10.312_{-0.239}^{+0.217}$ & $8.643 \pm 0.123$ \\
\hline PTF11qvh & 0.133 & $11042 \pm 12$ & $9.800_{-0.013}^{+0.171}$ & $\cdots$ \\
\hline PTF11rke & 0.094 & $12348 \pm 35$ & $9.968_{-0.136}^{+0.066}$ & $\cdots$ \\
\hline PTF11rpc & 0.143 & $11907 \pm 49$ & $11.315_{-0.183}^{+0.225}$ & $\ldots$ \\
\hline PTF11yp & 0.121 & $8812 \pm 41$ & $11.447_{-0.313}^{+0.068}$ & $\ldots$ \\
\hline PTF12awi & 0.045 & $9727 \pm 48$ & $10.503_{-0.108}^{+0.133}$ & $8.764 \pm 0.052$ \\
\hline PTF12cdb & 0.120 & $12068 \pm 20$ & $10.877_{-0.056}^{+0.212}$ & $\ldots$ \\
\hline PTF12cjg & 0.067 & $11080 \pm 17$ & $9.916_{-0.131}^{+0.105}$ & $8.615 \pm 0.142$ \\
\hline PTF12cyd & 0.170 & $11668 \pm 12$ & $9.118_{-0.073}^{+0.446}$ & $\ldots$ \\
\hline PTF12czu & 0.145 & $10709 \pm 151$ & $9.005_{-0.138}^{+0.021}$ & $\ldots$ \\
\hline PTF12dgy & 0.180 & $9911 \pm 23$ & $10.919_{-0.175}^{+0.367}$ & $\ldots$ \\
\hline PTF12dhb & 0.057 & $10701 \pm 12$ & $10.373_{-0.072}^{+0.030}$ & $8.618 \pm 0.032$ \\
\hline PTF12dhl & 0.057 & $10441 \pm 38$ & $11.001_{-0.012}^{+0.111}$ & $\ldots$ \\
\hline PTF12dhv & 0.140 & $12260 \pm 80$ & $10.442_{-0.160}^{+0.144}$ & $\cdots$ \\
\hline PTF12dst & 0.192 & $9385 \pm 24$ & $\begin{array}{c}9.939_{-0.019}^{+0.079} \\
\end{array}$ & $\ldots$ \\
\hline PTF12dwm & 0.053 & $9675 \pm 10$ & $10.538_{-0.200}^{+0.159}$ & $8.765 \pm 0.074$ \\
\hline PTF12dxm & 0.063 & $10505 \pm 9$ & $11.033_{-0.005}^{+0.005}$ & $\ldots$ \\
\hline PTF12egr & 0.132 & $10490 \pm 46$ & $11.520_{-0.013}^{+0.126}$ & $\ldots$ \\
\hline PTF12fhn & 0.125 & $10801 \pm 76$ & $11.281_{-0.011}^{+0.0143}$ & $\ldots$ \\
\hline PTF12fsd & 0.069 & $10862 \pm 25$ & $10.107_{-0.117}^{+0.098}$ & $8.398 \pm 0.063$ \\
\hline PTF12giy & 0.029 & $12093 \pm 177$ & $10.835_{-0.008}^{+0.177}$ & $8.826 \pm 0.096$ \\
\hline PTF12gnw & 0.100 & $10077 \pm 14$ & $8.816_{-0.234}^{+0.187}$ & $\ldots$ \\
\hline SN 1989M & 0.005 & $12330 \pm 50$ & $10.539_{-0.144}^{+0.627}$ & $\ldots$ \\
\hline SN 1994S & 0.015 & $10400 \pm 50$ & $11.113_{-0.520}^{+0.036}$ & $8.943 \pm 0.013$ \\
\hline SN 1997Y & 0.016 & $10510 \pm 50$ & $11.018_{-0.045}^{+0.067}$ & $9.023 \pm 0.012$ \\
\hline SN 1998dk & 0.013 & $12380 \pm 50$ & $9.982_{-0.186}^{+0.033}$ & $\cdots$ \\
\hline SN 1998es & 0.011 & $10240 \pm 50$ & $10.299_{-0.010}^{+0.052}$ & $\cdots$ \\
\hline SN 1999aa & 0.014 & $10350 \pm 50$ & $10.533_{-0.013}^{+0.132}$ & $9.035 \pm 0.027$ \\
\hline SN 1999ac & 0.009 & $10400 \pm 50$ & $10.254_{-0.128}^{+0.504}$ & $\ldots$ \\
\hline SN 1999da & 0.013 & $10660 \pm 50$ & $10.886_{-0.120}^{+0.039}$ & $\cdots$ \\
\hline SN 1999dq & 0.014 & $10860 \pm 50$ & $10.941_{-0.118}^{+0.422}$ & $\cdots$ \\
\hline SN 1999gd & 0.018 & $10420 \pm 50$ & $10.361_{-0.044}^{+0.168}$ & $\ldots$ \\
\hline SN 2000cp & 0.034 & $11000 \pm 50$ & $9.976_{-0.071}^{+0.459}$ & $8.841 \pm 0.026$ \\
\hline SN 2000dn & 0.032 & $10190 \pm 50$ & $11.081_{-0.177}^{+0.010}$ & $\ldots$ \\
\hline SN 2001bp & 0.095 & $10860 \pm 50$ & $10.602_{-0.165}^{+0.065}$ & $8.795 \pm 0.032$ \\
\hline SN 2001da & 0.017 & $11350 \pm 50$ & $10.633_{-0.185}^{+0.440}$ & $\ldots$ \\
\hline SN 2001ep & 0.013 & $10160 \pm 50$ & $10.215_{-0.117}^{+0.023}$ & $\cdots$ \\
\hline SN 2001fe & 0.014 & $11070 \pm 50$ & $10.341_{-0.077}^{+0.109}$ & $\ldots$ \\
\hline SN 2002aw & 0.026 & $10210 \pm 50$ & $10.931_{-0.076}^{+0.051}$ & $8.976 \pm 0.029$ \\
\hline SN 2002bf & 0.024 & $13680 \pm 50$ & $10.672_{-0.004}^{+0.004}$ & $\cdots$ \\
\hline SN 2002bo & 0.004 & $13070 \pm 50$ & $10.779_{-0.449}^{+0.054}$ & $\ldots$ \\
\hline SN 2002cd & 0.010 & $15230 \pm 50$ & $10.971_{-0.010}^{+0.124}$ & $\ldots$ \\
\hline SN 2002dk & 0.019 & $10430 \pm 50$ & $11.656_{-0.151}^{+0.048}$ & $\cdots$ \\
\hline SN 2002eb & 0.028 & $10230 \pm 50$ & $10.976_{-0.423}^{+0.164}$ & $\ldots$ \\
\hline SN 2002eu & 0.038 & $11020 \pm 50$ & $10.648_{-0.070}^{+0.493}$ & $\cdots$ \\
\hline SN 2002ha & 0.014 & $10930 \pm 50$ & $11.075_{-0.198}^{+0.481}$ & $\ldots$ \\
\hline SN 2003U & 0.026 & $11300 \pm 50$ & $9.670_{-0.377}^{+0.614}$ & $8.828 \pm 0.021$ \\
\hline SN 2003Y & 0.017 & $9860 \pm 50$ & $10.728_{-0.037}^{+0.153}$ & $\cdots$ \\
\hline SN 2003cq & 0.033 & $12080 \pm 50$ & $11.884_{-0.111}^{+0.064}$ & $\cdots$ \\
\hline SN 2003he & 0.025 & $11310 \pm 50$ & $10.335_{-0.069}^{+0.545}$ & $\ldots$ \\
\hline SN $2004 \mathrm{dt}$ & 0.020 & $13540 \pm 50$ & $10.875_{-0.144}^{+0.020}$ & $\cdots$ \\
\hline SN 2004gs & 0.027 & $10430 \pm 50$ & $10.740_{-0.032}^{+0.137}$ & $\ldots$ \\
\hline SN 2005M & 0.022 & $10670 \pm 60$ & $9.898_{-0.030}^{+0.081}$ & $8.504 \pm 0.006$ \\
\hline SN 2005W & 0.009 & $10600 \pm 50$ & $10.473_{-0.154}^{+0.007}$ & $\ldots$ \\
\hline SN 2005ao & 0.038 & $11460 \pm 50$ & $10.915_{-0.033}^{+0.161}$ & $\ldots$ \\
\hline SN 2005ag & 0.079 & $11370 \pm 50$ & $\ldots$ & $9.250 \pm 0.021$ \\
\hline SN 2005bc & 0.012 & $10700 \pm 50$ & $10.383_{-0.120}^{+0.018}$ & $9.054 \pm 0.007$ \\
\hline
\end{tabular}


TABLE 4

SuMMARY OF OUR SAMPLE IN THIS WORK (CONTINUED).

\begin{tabular}{|c|c|c|c|c|}
\hline SN Name & Redshift & $\begin{array}{c}v_{\mathrm{SiII}} \\
\left(\mathrm{km} \mathrm{s}^{-1}\right)\end{array}$ & $\begin{array}{c}\log M_{\text {stellar }} \\
\left(\mathrm{M}_{\odot}\right)\end{array}$ & $12+\log (\mathrm{O} / \mathrm{H})$ \\
\hline SN $2005 \mathrm{cg}$ & 0.032 & $11560 \pm 50$ & $8.574_{-0.025}^{+0.057}$ & $7.947 \pm 0.039$ \\
\hline SN 2005er & 0.026 & $8740 \pm 50$ & $11.478_{-0.114}^{+0.492}$ & $\ldots$ \\
\hline SN 2005eq & 0.029 & $10090 \pm 50$ & $11.424_{-0.452}^{+0.029}$ & $\cdots$ \\
\hline SN 2005hj & 0.058 & $10550 \pm 50$ & $10.046_{-0.038}^{+0.078}$ & $8.519 \pm 0.038$ \\
\hline SN 2005ki & 0.020 & $11030 \pm 50$ & $11.146_{-0.120}^{+0.035}$ & $\ldots$ \\
\hline SN $2005 \mathrm{~ms}$ & 0.025 & $11840 \pm 50$ & $10.684_{-0.054}^{+0.210}$ & $\cdots$ \\
\hline SN 2006N & 0.014 & $11300 \pm 50$ & $10.545_{-0.143}^{+0.494}$ & $\ldots$ \\
\hline SN 2006S & 0.032 & $10710 \pm 50$ & $10.628_{-0.184}^{+0.006}$ & $\cdots$ \\
\hline SN 2006bt & 0.032 & $10510 \pm 50$ & $11.124_{-0.155}^{+0.027}$ & $\cdots$ \\
\hline SN 2006bz & 0.028 & $10850 \pm 50$ & $10.471_{-0.020}^{+0.006}$ & $\cdots$ \\
\hline SN $2006 \mathrm{~cm}$ & 0.016 & $11150 \pm 50$ & $10.913_{-0.047}^{+0.069}$ & $\cdots$ \\
\hline SN 2006cq & 0.048 & $10160 \pm 50$ & $11.212_{-0.0141}^{+0.011}$ & $\ldots$ \\
\hline SN 2006cs & 0.024 & $10730 \pm 50$ & $10.913_{-0.024}^{+0.490}$ & $\ldots$ \\
\hline SN 2006or & 0.021 & $11340 \pm 50$ & $11.114_{-0.117}^{+0.034}$ & $\cdots$ \\
\hline SN 2006sr & 0.024 & $12470 \pm 50$ & $10.778_{-0.163}^{+0.325}$ & $\cdots$ \\
\hline SN 2007A & 0.018 & $10600 \pm 50$ & $10.775_{-0.241}^{+0.252}$ & $\cdots$ \\
\hline SN $2007 \mathrm{~N}$ & 0.013 & $10330 \pm 50$ & $10.376_{-0.141}^{+0.017}$ & $\cdots$ \\
\hline SN $2007 \mathrm{O}$ & 0.036 & $10000 \pm 50$ & $11.033_{-0.117}^{+0.007}$ & $9.009 \pm 0.011$ \\
\hline SN 2007af & 0.005 & $10560 \pm 50$ & $9.562_{-0.096}^{+0.181}$ & $\ldots$ \\
\hline SN 2007ba & 0.038 & $9630 \pm 50$ & $11.118_{-0.068}^{+0.105}$ & $\cdots$ \\
\hline SN 2007bc & 0.021 & $9850 \pm 50$ & $10.922_{-0.000}^{+0.020}$ & $\ldots$ \\
\hline SN 2007bz & 0.022 & $11700 \pm 50$ & $9.504_{-0.100}^{+0.085}$ & $8.329 \pm 0.012$ \\
\hline SN 2007ci & 0.018 & $11830 \pm 50$ & $10.969_{-0.150}^{+0.007}$ & $\ldots$ \\
\hline SN 2007fr & 0.051 & $10740 \pm 50$ & $11.770_{-0.102}^{+0.032}$ & $\cdots$ \\
\hline SN 2007gi & 0.005 & $14870 \pm 50$ & $10.643_{-0.151}^{+0.47 \overline{9}}$ & $\cdots$ \\
\hline SN 2007hj & 0.014 & $11710 \pm 50$ & $10.580_{-0.143}^{+0.484}$ & $\cdots$ \\
\hline SN 2007s1 & 0.027 & $11370 \pm 50$ & $9.998_{-0.030}^{+0.143}$ & . \\
\hline SN 2008Z & 0.021 & $11460 \pm 50$ & $9.488_{-0.064}^{+0.203}$ & $8.359 \pm 0.060$ \\
\hline SN 2008ar & 0.026 & $10340 \pm 50$ & $10.880_{-0.512}^{+0.071}$ & $8.972 \pm 0.016$ \\
\hline SN 2008s1 & 0.022 & $10560 \pm 50$ & $10.559_{-0.040}^{+0.129}$ & $\ldots$ \\
\hline SN 2008dx & 0.023 & $8850 \pm 50$ & $10.678_{-0.136}^{+0.027}$ & $\cdots$ \\
\hline SN 2008ec & 0.016 & $10750 \pm 50$ & $10.930_{-0.075}^{+0.124}$ & $\ldots$ \\
\hline SN 2009an & 0.009 & $12680 \pm 50$ & $10.981_{-0.000}^{+0.084}$ & $9.086 \pm 0.014$ \\
\hline SN 2009fx & 0.048 & $10110 \pm 50$ & $10.000_{-0.170}^{+0.020}$ & $\cdots$ \\
\hline SN 2009ig & 0.009 & $13660 \pm 50$ & $10.454_{-0.175}^{+0.000}$ & $\ldots$ \\
\hline SN 2009no & 0.046 & $10030 \pm 50$ & $10.319_{-0.187}^{+0.041}$ & $8.776 \pm 0.028$ \\
\hline SN 2010ex & 0.023 & $10890 \pm 50$ & $10.089_{-0}^{+0}$ & $\cdots$ \\
\hline SN 2010ii & 0.027 & $12240 \pm 50$ & $11.142_{-0.1}^{+0.0}$ & $\ldots$ \\
\hline SN 2010iw & 0.022 & $10360 \pm 50$ & $9.731_{-0.092}^{+0.066}$ & $\ldots$ \\
\hline SN 2011ao & 0.011 & $10340 \pm 50$ & $9.877_{-0.032}^{+0.141}$ & $8.481 \pm 0.010$ \\
\hline SN 2011by & 0.003 & $10270 \pm 50$ & $9.354_{-0.191}^{+0.014}$ & $8.624 \pm 0.022$ \\
\hline SN 2011hb & 0.029 & $11800 \pm 50$ & $11.247_{-0.051}^{+0.004}$ & $\ldots$ \\
\hline SN 2011ia & 0.017 & $10680 \pm 50$ & & $8.865 \pm 0.010$ \\
\hline SN 2012bh & 0.025 & $10360 \pm 50$ & $10.538_{-0.187}^{+0.406}$ & $\ldots$ \\
\hline SN 2012cg & 0.001 & $10580 \pm 50$ & $8.467_{-0.160}^{+0.035}$ & $8.729 \pm 0.007$ \\
\hline SN 2012da & 0.018 & $11110 \pm 50$ & $9.580_{-0.0}^{+0.0}$ & $\cdots$ \\
\hline SN 2013di & 0.024 & $11460 \pm 50$ & $11.008_{-0.060}^{+0.530}$ & $\cdots$ \\
\hline
\end{tabular}

